\title{
Tumor neuroendocrino de colon derecho en un paciente con colitis ulcerativa: a propósito de un caso
}

\section{Right colon neuroendocrine tumor in a patient with ulcerative colitis: A case study}

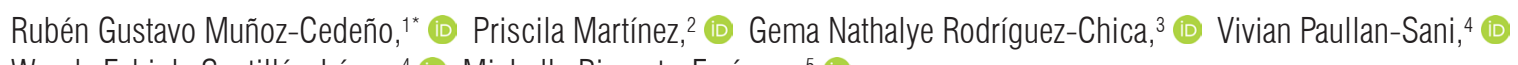
Wendy Fabiola Santillán-López, ${ }^{4}$ (D) Michelle Ricaurte-Enríquez. ${ }^{5}$

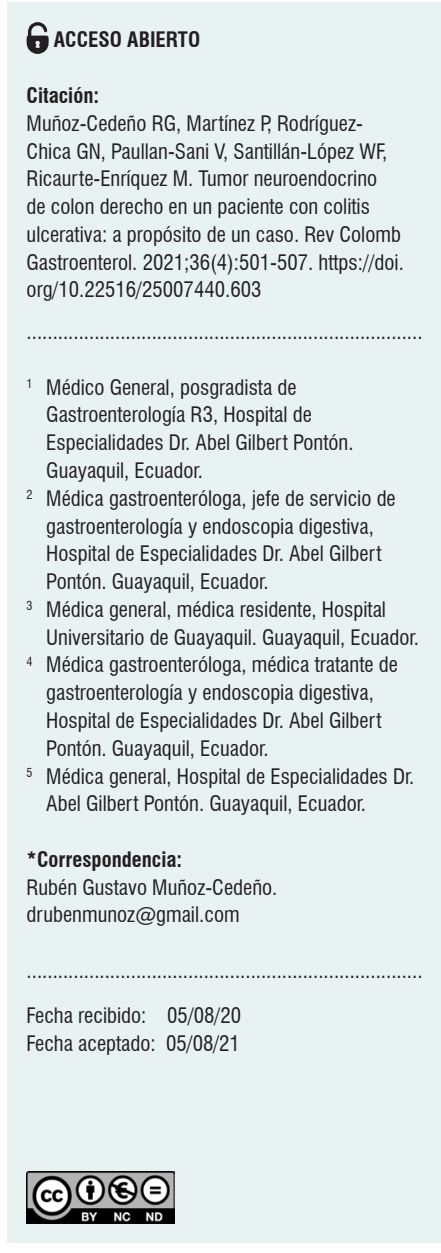

\section{Resumen}

Los tumores neuroendocrinos del colon son relativamente raros en comparación con el carcinoma colorrectal esporádico; su incidencia es muy baja y la localización más frecuente es en el ciego, colon sigmoideo y recto. Se describe con poca frecuencia la asociación de tumor neuroendocrino con la colitis ulcerativa debido a la inflamación crónica que conduce a la diferenciación de células neuroendocrinas a partir de células multipotenciales en el epitelio displásico, que puede ser responsable del desarrollo de carcinomas neuroendocrinos colorrectales. La presentación del caso se trata de un paciente de 57 años de edad con colitis ulcerativa con cuadro de dolor abdominal, pérdida de peso y diarreas líquidas con moco. En el examen físico presentó una lesión endurecida en el marco colónico derecho con tomografía de lesión neoplásica en el colon ascendente y en su hospitalización presentó un cuadro de oclusión intestinal, por lo que se le realizó una hemicolectomía más ileostomía, cuyo resultado en la biopsia fue un tumor neuroendocrino G3 de células grandes en el colon ascendente. En este artículo se revisan de forma práctica los aspectos relacionados con la fisiopatología, diagnóstico y tratamiento de la asociación de estas dos patologías.

\section{Palabras clave}

Tumor neuroendocrino gastrointestinal, colitis ulcerativa, enfermedad inflamatoria intestinal, tumor de colon neuroendocrino.

\section{Abstract}

Neuroendocrine colon tumors are relatively unknown compared with sporadic colorectal cancer; its incidence is low, and it is generally located in the cecum, sigmoid colon, and rectum. The existing relation between neuroendocrine tumors and ulcerative colitis is not frequently described due to the chronic inflammation that leads to neuroendocrine cell differentiation from multipotential cells in the dysplastic epithelium, which can be responsible for colorectal neuroendocrine carcinomas development. The study refers to the case of a patient of 57 years old with ulcerative colitis, abdominal pain, weight loss, and liquid diarrhea with mucus. Physical examination revealed a hardened lesion in the right colonic framework with the tomography of a neoplastic lesion in the ascending colon. When the patient was hospitalized, he developed an intestinal obstruction. A hemicolectomy plus ileostomy procedure was performed resulting in a large cell G3 neuroendocrine tumor in the ascending colon revealed in the biopsy. In this article, the aspects related to the pathophysiology, diagnosis, and treatment of the association of these two pathologies are reviewed in a practical way.

\section{Keywords}

Gastrointestinal neuroendocrine tumor, Ulcerative colitis, Inflammatory bowel disease, Neuroendocrine colon tumors. 


\section{INTRODUCCIÓN}

Los tumores neuroendocrinos gastrointestinales son infrecuentes, derivados de células neuroendocrinas, distribuidas principalmente en la mucosa y submucosa del tracto gastrointestinal, su frecuencia anual es de 8,4/100 000 habitantes, representa el 2,5\% de las neoplasias gastrointestinales y el $0,49 \%$ de todos los tumores en general, predomina entre la sexta y séptima décadas de la vida y las localizaciones más frecuentes son el ciego, colon sigmoideo y recto ${ }^{(1)}$.

Los tumores neuroendocrinos del colon son relativamente raros en comparación con el carcinoma colorrectal esporádico, su incidencia es muy baja (del 0,6 \%) y existen pocos informes de tumores neuroendocrinos de colon y recto en pacientes con colitis ulcerativa. La prevalencia de los tumores neuroendocrinos es difícil determinar y no se ha establecido una relación definitiva entre estos tumores y la enfermedad inflamatoria intestinal ${ }^{(2)}$.

\section{PRESENTACIÓN DEL CASO}

Se trata de un paciente masculino de 57 años de edad con antecedentes patológicos personales de colitis ulcerativa diagnosticado hace 2 años con tratamiento de mesalazina $3 \mathrm{~g}$ cada día y antecedentes familiares de hermano con colitis ulcerativa con tratamiento de mesalazina. Presentó un cuadro clínico de inicio de 2 años de evolución por dolor abdominal generalizado, pérdida de peso de $20 \mathrm{~kg}$ aproximadamente, diarreas líquidas con moco y distensión abdominal. Ingresó al centro de salud por una agudización de su cuadro clínico hace 15 días con dolor abdominal tipo cólico acompañado con distensión abdominal, diarrea líquida con moco y artralgia sin artritis; además, mencionó la pérdida de peso aproximado en 1 año de $10 \mathrm{~kg}$.

Al examen físico se encontró abdomen blando depresible y se palpó un endurecimiento en el flanco y la fosa ilíaca derecha. En los exámenes de ingreso se reportó hemoglobina de $6,0 \mathrm{mg} / \mathrm{dL}$ y hematocrito de $21,50 \%$; leucocitos de $13300 / \mathrm{mm}^{3}$ con neutrófilos de $83,7 \%$, plaquetas de 1 257 000, proteína C-reactiva (PCR) de $8,20 \mathrm{mg} / \mathrm{dL}$, albúmina de $3 \mathrm{mg} / \mathrm{dL}$, proteínas totales de 5,80 , electrolitos y función renal normales; laboratorio de investigación de enfermedades venéreas (VDRL) no reactivo; vitamina $B_{12}$ $>$ 1000; ferritina de 90,30; velocidad de sedimentación glomerular (VSG) de 30; hierro sérico de 31; transferrina de 205; marcadores tumorales dentro de los valores normales; prueba cutánea de tuberculina o prueba de Mantoux negativa; serología para citomegalovirus (CMV), virus de herpes, rubéola, virus de hepatitis B (VHB), virus de hepatitis $\mathrm{C}(\mathrm{VHC})$ y virus de inmunodeficiencia humana (VIH) negativos; coproparasitario y toxina de Clostridium difficile negativos para antígeno; cultivo de heces negativo y sangre oculta positivo. Se reportó un índice de Truelove-Witts de 19 puntos con brote moderado.

La ecografía abdominal reportó que a nivel del flanco derecho se observa un asa intestinal de paredes engrosadas irregulares de aspecto tumoral de $79 \times 78 \mathrm{~mm}$ con ecografía Doppler e incremento de la vascularización. En la tomografía axial computarizada (TAC) de abdomen y pelvis se observa un engrosamiento difuso de las paredes del ángulo hepático hasta el colon ascendente y ciego con alteración de la grasa pericecal, y cambios inflamatorios en el íleon terminal, por lo que se debe descartar lesión primaria en el ciego (Figuras 1 y 2). Se realizó un estudio endoscópico digestivo alto en el que se observó una pangastropatía atrófica; la colonoscopia reveló que en todo el colon se observó alteración del patrón vascular, eritema, granular, edematosa, friable, a nivel del ángulo hepático una estenosis de aspecto maligno que impide el paso del equipo con un reporte colonoscópico de pancolitis activa con un puntaje de Mayo 2 para colitis ulcerativa. Se tomó una biopsia de la estenosis y de todos los segmentos y se reportó infiltrado inflamatorio grave de linfocitos y células plasmáticas, neutrófilos en la lámina propia, neutrófilos en el epitelio, daño epitelial, daño en las criptas de la superficie, criptitis, absceso críptico agregados linfoides, ulceración superficial y profunda, y pérdida de algunas células caliciformes sugestivos de colitis ulcerativa; y en el resto de los segmentos no se observaron granulomas sugestivos de colitis ulcerativa.

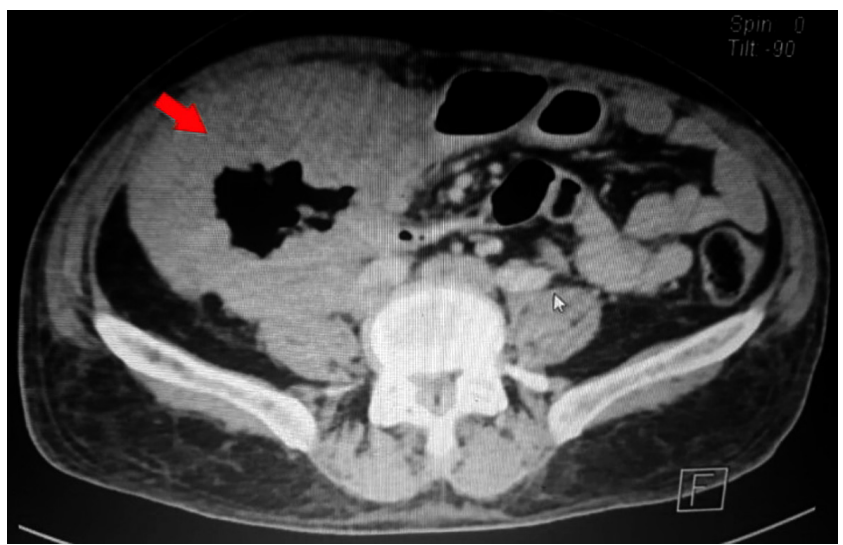

Figura 1. TAC de abdomen en corte transversal. Se observa un engrosamiento de la pared del colon derecho, como se observa en la flecha roja.

Paciente con evolución tórpida con un índice de TrueloveWitts de 19 puntos con brote moderado, colonoscopia con puntaje Mayo 2, presentó un cuadro de dolor abdominal de moderada a gran intensidad en la escala visual análoga (EVA) de 8/10, distensión abdominal, estreñimiento y vómitos posprandiales sugestivos de cuadro de oclusión intestinal. Se realizó una radiografía de abdomen en la que 
se observaron niveles hidroaéreos a nivel del colon transverso (Figura 3).

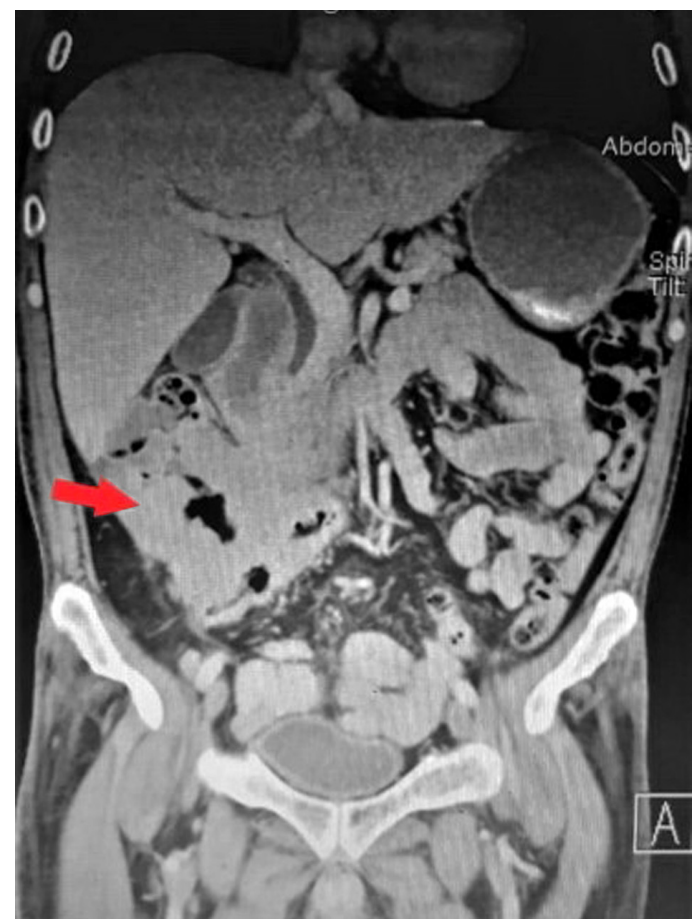

Figura 2. TAC de abdomen en corte coronal en la que se observa un engrosamiento de la pared del colon derecho con alteración de la grasa pericecal, como se observa en la flecha roja.
Se solicitó valoración por el servicio de coloproctología y se decidió la conducta quirúrgica en la que se le realizó una colectomía total más ileostomía con hallazgos quirúrgicos de tumor de ciego que involucra el colon sigmoide e íleon terminal, con una biopsia que reportó una hemicolectomía total con íleon terminal que mide $45 \times 4 \times 4 \mathrm{~cm}$ de superficie lisa cubierto de moderada cantidad de tejido adiposo; al corte hubo consistencia elástica. Se observó mucosa de pliegues edematizados y válvula ileocecal obstruida a nivel del borde quirúrgico; se observó una lesión sobreelevada que mide $40 \times 30 \mathrm{~mm}$ indurada que dista a $25 \mathrm{~mm}$ del borde marcado con sutura; colon ascendente deformado que mide $20 \times 8 \times 5 \mathrm{~cm}$ desde el ciego hasta el borde quirúrgico, de superficie lisa y color café violáceo; al corte hubo consistencia elástica. Se observó a nivel del ciego una lesión sobreelevada de bordes mal definidos que dista de $45 \mathrm{~cm}$ del borde quirúrgico ileal y $14 \mathrm{~cm}$ del borde quirúrgico colónico; midió 12 x 10 x 10 cm; ocupó el $100 \%$ de la circunferencia intestinal y obstruyó el $100 \%$ de la luz colónica, dista a $4 \mathrm{~cm}$ del borde quirúrgico mesentérico y dio como resultado una carcinoma poco diferenciado G3 con rasgos neuroendocrinos de células grandes, ulcerado, multicéntrico, ubicado en 2 focos (ciego e íleon) con un recuento mitótico de más de 20 mitosis en 10 campos de gran aumento. No se identificó perforación transmural macroscópica ni microscópica, evidencia de infiltración linfovascular y perineural, bordes quirúrgicos colónicos ileal y mesentéricos libres, con 5 ganglios linfáticos aisla-
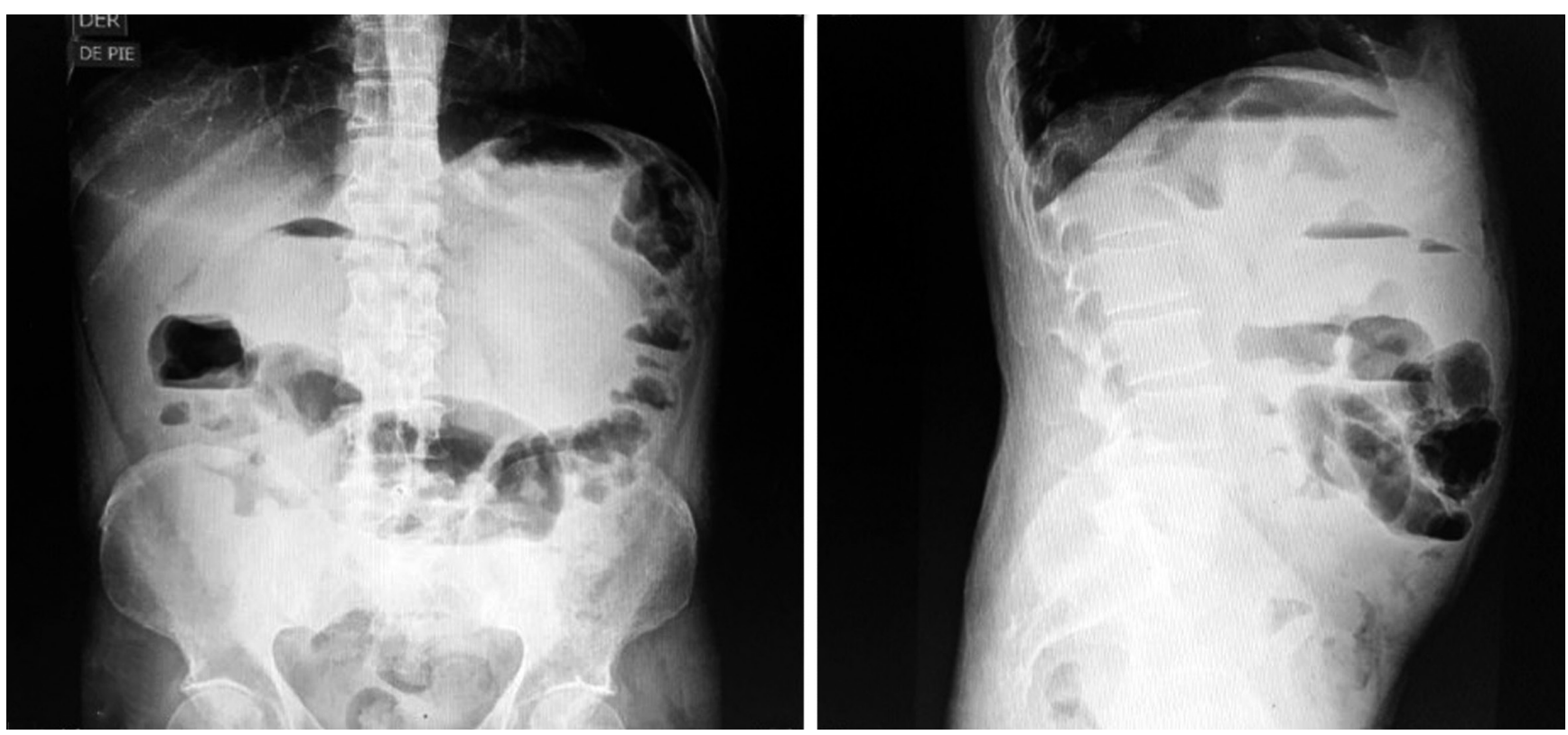

Figura 3. Radiografía abdomen simple de pie en la que se evidencian niveles hidroaéreos, sugestivos de obstrucción intestinal. 
dos, pericólicos libres de lesión e inmunohistoquímicas de diferenciación neuroendocrina.

El posquirúrgico fue manejado por un equipo multidisciplinario con buena evolución y fue dado de alta médica sin complicaciones con controles en consulta externa. El paciente no acudió a las consultas subsecuentes y las curaciones de la herida quirúrgica, en las que presentó complicaciones posteriores a la cirugía con infección de sitio quirúrgico con cuadro de síndrome de respuesta inflamatoria, por lo que ingresó en malas condiciones clínicas con leucocitosis, neutrofilia, taquicardia, hipotensión, alza térmica con hemocultivo de Staphylococcus epidermidis, sensible a clindamicina, linezolid y vancomicina. Pasó a terapia intensiva, en la que el paciente tuvo una evolución desfavorable y falleció.

\section{DISCUSIÓN}

La incidencia de tumores neuroendocrinos gastrointestinales es de 0,1 \% y 3,9 \% de todas las neoplasias colorrectales. En el Instituto Nacional de Cáncer Estadounidense es de solo el 0,3\% de los cánceres colorrectales que serían de tipo neuroendocrino y el Centro de Cáncer Sloan-kettering Memorial de Nueva York informó una incidencia del 0,6 \% de carcinomas neuroendocrinos y solo el $0,2 \%$ de carcinoma neuroendocrino colorrectal de células grandes. Son tumores poco frecuentes y se describen con poca frecuencia en el contexto de la enfermedad inflamatoria intestinal en el colon y recto ${ }^{(3,4)}$.

Los tumores carcinoides y los neuroendocrinos también pueden estar asociados con enfermedad inflamatoria intestinal del colon en función a los hallazgos de un número mayor de células neuroendocrinas en la mucosa inflamada, lo que sugiere que la inflamación prolongada es directamente responsable del desarrollo de esta enfermedad ${ }^{(5)}$.

La colitis ulcerativa tiene un mayor riesgo conocido para desarrollar carcinoma colorrectal y displasia, el riesgo aumenta con la duración y la extensión anatómica de la enfermedad. Un metaanálisis realizado por Eaden y colaboradores en 2001 estimó que la prevalencia general de cáncer colorrectal en cualquier paciente con colitis ulcerativa es del $3,7 \%$, con un riesgo de carcinoma colorrectal del $2 \%$ en 10 años, del $8 \%$ en 20 años y del $18 \%$ en 30 años de la enfermedad; con base en esto se establecieron pautas específicas para el cribado y la vigilancia del carcinoma colorrectal en pacientes con colitis ulcerativa para detectar la neoplasia en una etapa curable quirúrgicamente y para reducir la mortalidad relacionada con el carcinoma colorrectal ${ }^{(3,6)}$.

La mayoría de los carcinomas colorrectales asociados con la enfermedad inflamatoria intestinal es histológicamente similar al tipo esporádico y los tumores con características neuroendocrinas son muy inusuales. La displasia por infla- mación crónica que conduce a la diferenciación de células neuroendocrinas puede ser responsable del desarrollo de carcinomas neuroendocrinos colorrectales ${ }^{(7,8)}$.

En un estudio de Sigel y colaboradores se informó que la diferenciación neuroendocrina podría evolucionar a partir de células multipotenciales en el epitelio displásico, lo que sugiere que la inflamación de larga data podría estar involucrada por su patogénesis. En cuanto a los tumores neuroendocrinos que no sean carcinoides, en 14 pacientes con enfermedad de intestino irritable (EII) se observó que todos los tumores surgieron en áreas afectadas por la EII, 8 pacientes con enfermedad de Crohn y 6 con colitis ulcerativa. 6 de las 14 neoplasias afectaron al recto. Los tumores neuroendocrinos estaban bien diferenciados en 11 casos y mal diferenciadas en 3 casos; 2 de estos 3 pacientes murieron a los 3 y 11 meses después de la escisión del tumor ${ }^{(9)}$. Shigaki y colaboradores observaron hallazgos similares en 2013 , con un $30 \%$ a $50 \%$ de las neoplasias asociadas a colitis ulcerativa que muestran características de diferenciación neuroendocrina ${ }^{(10)}$.

Las características clinicopatológicas de estos pacientes son alteración del ritmo evacuatorio, diarreas crónicas, hematoquecia, sangrado oscuro evidente, dolor abdominal, síntomas inespecíficos y en ocasiones cuadros obstructivos. El síndrome carcinoide es raro, se observa en menos del $5 \%$ de los $\operatorname{casos}^{(11,12)}$.

La TAC y la resonancia magnética (RM) tienen mayor sensibilidad para establecer compromisos de invasión, pero la resonancia es más sensible y específica para establecer la invasión de la lesión a la muscular propia o sospecha de compromiso ganglionar. Actualmente, la tomografía por emisión de positrones (PET-CT) con galio $68\left({ }^{68} \mathrm{Ga}\right)$-Dotatate se considera la imagen de elección para el diagnóstico, estadificación y seguimiento de los tumores neuroendocrinos, técnica que permite mostrar la imagen de fusión y la sobreexpresión local de los receptores de somatostatina.

Los tumores neuroendocrinos de colon son neoplasias raras y agresivas caracterizadas por un crecimiento organoide y difuso de altas células neoplásicas atípicas, pequeñas y grandes, con más de 20 figuras mitóticas por 10 campos de alta potencia $(\times 400)$, un Ki-67 índice proliferativo mayor del $20 \%$ y grandes áreas de necrosis geográfica; las células neoplásicas muestran características morfológicas e inmunohistoquímicas de diferenciación neuroendocrina ${ }^{(13)}$. Es impórtate diferenciar los carcinomas neuroendocrinos de los adenocarcinomas de colon y recto patológicamente porque los pacientes pueden beneficiarse de regímenes quimioterapéuticos citotóxicos alternativos ${ }^{(14)}$.

La diferenciación en la carcinogénesis relacionada con la EII fue demostrada por Shigaki y colaboradores, utilizando inmunohistoquímica para p53 y cromogranina A, se observó que un aumento de células neuroendocrinas 
estuvo presente desde las primeras etapas de las lesiones preneoplásicas en EII (bajo grado de displasia), a través de displasia de alto grado, hasta que se relacione la EII con un adenocarcinoma invasivo ${ }^{(10)}$.

En el diagnóstico de los tumores neuroendocrinos, solo el $45 \%$ de ellos está limitado localmente, los ubicados en el colon habitualmente se presentan como grandes masas tumorales y, de acuerdo con una revisión sistemática, tienen el peor pronóstico; los ubicados en el recto son diagnosticados precozmente en la submucosa, son de aspecto polipoide y están cubiertos por mucosa glandular. Los menos frecuentes están en el colon distal y recto, en el $25 \%$ de ellos predomina la variedad de células pequeñas, a veces asociada con adenocarcinoma o carcinoma de células escamosas. Un $16 \%$ y $40 \%$ presentan metástasis a distancia ${ }^{(1,11)}$.

Las recomendaciones del tratamiento quirúrgico de las neoplasias neuroendocrinas del colon son similares a las del tratamiento para el adenocarcinoma de colon: en los pacientes sin metástasis a distancia se realiza la resección más linfadenectomía local (por laparoscopia o a cielo abierto); en los tumores con metástasis a distancia, resección paliativa más linfadenectomía regional, con quimioterapia previa para reducir la lesión; en los casos en que la resección no haya sido completa o haya habido invasión a órganos adyacentes, hemicolectomía derecha, izquierda o transversa, dependiendo del compromiso del drenaje linfático observado en la cirugía ${ }^{(15,16)}$.

El tratamiento de tumores neuroendocrinos es totalmente quirúrgico, y los tratamientos coadyuvantes, como la quimioterapia o radioterapia, son un factor menor para mejorar la supervivencia del paciente. Se desarrollan ciclos de quimioterapia, como es el caso de algunos esquemas agresivos, basados en la administración de estreptozotocina y 5-fluorouracilo o de doxorrubicina con 5-fluorouracilo. La radioterapia está indicada en las metástasis óseas o del sistema nervioso central. Los tumores neuroendocrinos de colon y recto son raros, agresivos con rápida tendencia a la invasión locorregional y a distancia, y la cirugía es el único tratamiento efectivo, aunque se están desarrollando nuevas líneas de quimioterapia ${ }^{(17,18)}$.

El tratamiento quirúrgico en pacientes con colitis ulcerativa es de aproximadamente $20 \%$ a $40 \%$ de los pacientes en algún momento de la vida, por presentar una afectación grave en la evolución de su enfermedad y está indicado como electivo en $>90 \%$ por fracaso del tratamiento médico, profilaxis o tratamiento por displasia de alto grado, displasia de bajo grado o asociado con cáncer colorrectal, o como de urgencia en $<10 \%$ por causas de perforación, hemorragia masiva, obstrucción y megacolon tóxico que no responde al tratamiento médico en 72 horas ${ }^{(19)}$.
Existe controversia sobre el manejo del muñón rectal, y se ha aceptado tanto el cierre distal mediante el procedimiento de Hartmann como la creación de una fístula mucosa, en la que la colocación extrafascial del segmento rectosigmoideo distal puede estar asociada con menores complicaciones pélvicas sépticas por estar cerrado el muñón distal. Actualmente, la proctocolectomía restauradora con reservorio ileoanal es considerada el estándar de oro para el tratamiento de colitis ulcerativa ${ }^{(20)}$.

La anastomosis ileorrectal es a menudo una operación difícil que se realiza en segundo plano, ya que se debe movilizar la ileostomía para efectuar la unión con el extremo superior del recto. También existen revisiones acerca de las complicaciones asociadas con la implementación de reservorio ileal (mecánicas, inflamatorias, infecciosas, funcionales, neoplásicas y metabólicas) ${ }^{(21)}$.

El uso tópico preoperatorio de corticoides y mesalazina hace seguro conservar el recto para realizar ileoproctostomía, pero se ha demostrado en estudios que el uso de la terapia tópica de mesalazina y corticoide posoperatorio diario por un período indefinido realizados en el hogar de los pacientes previene la recurrencia de la proctitis y da como resultado una función aceptable de la anastomosis ileorrectal ${ }^{(22)}$.

La complicación más frecuente es la reservoritis, especialmente importante por su elevada frecuencia en la evolución y cuyo tratamiento varía en función de la respuesta y su curso evolutivo, que puede ser manejada con un enema de budesonida o con supositorios de mesalazina para evitar complicaciones de pouchitis. Uno de los problemas de la colectomía es el incremento de la frecuencia de las deposiciones, propiciado, en gran medida, por la pérdida de una función importante del colon, como absorción de agua y electrolitos ${ }^{(21)}$.

Las medidas paliativas como la cirugía, la confección de colostomías o los tratamientos radioterápicos deben ser evaluados para mejorar la calidad de vida del paciente con estos tumores ${ }^{(18)}$. En las enfermedades metastásicas existen tratamientos alternativos o adjuntos que pueden emplearse, como la ablación con radiofrecuencia, la crioterapia y la quimioembolización transarterial ${ }^{(23)}$.

La supervivencia a los 5 años oscila entre el $40 \%$ y $70 \%$, según los parámetros de grado histológico, índice de proliferación celular y estadio clínico; por tanto, corresponden a tumores neuroendocrinos bien diferenciados G3 y a carcinomas neuroendocrinos pobremente diferenciados ${ }^{(24)}$.

Los tumores están pobremente diferenciados, son extremadamente agresivos y tienen un pronóstico desfavorable, con aproximadamente el $70 \%$ de los pacientes que presentan enfermedad metastásica y una tasa de supervivencia media reportada de entre el 5 y 11 meses con tasas de supervivencia a un año entre el $10 \%$ y $15 \%^{(14,25)}$. 


\section{CONCLUSIONES}

Los tumores neuroendocrinos asociados con colitis ulcerativa son relativamente raros y existen pocos informes de tumores neuroendocrinos de colon, esto se debe a la inflamación crónica que conduce a la diferenciación de células neuroendocrinas a partir de células multipotenciales en el epitelio displásico en pacientes de larga data con colitis ulcerativa, a diferencia de nuestro caso, en el que el paciente no tenía muchos años con el diagnóstico, lo que nos hace pensar que falta más investigación o reporte de esta asociación. Las revisiones sistemáticas indican que los tumores neuroendocrinos pobremente diferenciados y de células grandes son de peor pronóstico, con una tasa de supervivencia a un año muy baja.

\section{Conflicto de intereses}

No existen conflictos de intereses.

\section{Fuente de financiación}

Propias de los autores.

\section{REFERENCIAS}

1. Bannura G, Barrera A, Melo C, Illanes F, Gallardo C. Tumores neuroendocrinos primarios de colon y recto. Rev Chil Cir. 2018;70(1):53-58. https://doi.org/10.4067/S0718-40262018000100053

2. Stoner P, Ghaffaripour T, Cohen D. Aggressive largecell neuroendocrine carcinoma of the sigmoid colon in a patient with ulcerative colitis. BMJ Case Rep. 2017;2017:bcr2017220093.

https://doi.org/10.1136/bcr-2017-220093

3. Grassia R, Bodini P, Dizioli P, Staiano T, Iiritano E, Bianchi G, Buffoli F. Neuroendocrine carcinomas arising in ulcerative colitis: coincidences or possible correlations? World J Gastroenterol. 2009;15(33):4193-5. https://doi.org/10.3748/wjg.15.4193

4. Vilallonga R, Espín Basany E, López Cano M, Landolfi S, Armengol Carrasco M. Carcinomas neuroendocrinos de colon y recto. Experiencia de una unidad en seis años. Rev Esp Enferm Dig. 2008;100(1):11-6. https://doi.org/10.4321/s1130-01082008000100003

5. Wong NA, Harrison DJ. Colorectal neoplasia in ulcerative colitis-recent advances. Histopathology. 2001;39(3):221-34. https://doi.org/10.1046/j.1365-2559.2001.01292.x

6. Eaden JA, Abrams KR, Mayberry JF. The risk of colorectal cancer in ulcerative colitis: a meta-analysis. Gut. 2001;48(4):526-35.

https://doi.org/10.1136/gut.48.4.526

7. Carvalho JR, Fernandes SR, Correia LA. Extensive Neuroendocrine Adenocarcinoma in Ulcerative Colitis. J Crohns Colitis. 2016;10(1):116-7. https://doi.org/10.1093/ecco-jcc/jjv177

8. Rifu K, Koinuma K, Horie H, Morimoto M, Kono Y, Tahara M, Sakuma Y, Hosoya Y, Kitayama J, Lefor AK, Sata N, Suzuki T, Fukushima N. Sigmoid colon carcinoma with focal neuroendocrine differentiation associated with ulcerative colitis: A case report. Int J Surg Case Rep. 2016;23:151-6.

https://doi.org/10.1016/j.ijscr.2016.04.035
9. Sigel JE, Goldblum JR. Neuroendocrine neoplasms arising in inflammatory bowel disease: a report of 14 cases. Mod Pathol. 1998;11(6):537-42.

10. Shigaki K, Mitomi H, Fujimori T, Ichikawa K, Tomita S, Imura J, Fujii S, Itabashi M, Kameoka S, Sahara R, Takenoshita S. Immunohistochemical analysis of chromogranin A and p53 expressions in ulcerative colitisassociated neoplasia: neuroendocrine differentiation as an early event in the colitis-neoplasia sequence. Hum Pathol. 2013;44(11):2393-9. https://doi.org/10.1016/j.humpath.2013.06.008

11. Korse CM, Taal BG, van Velthuysen ML, Visser O. Incidence and survival of neuroendocrine tumours in the Netherlands according to histological grade: experience of two decades of cancer registry. Eur J Cancer. 2013;49(8):1975-83. https://doi.org/10.1016/j.ejca.2012.12.022

12. Shia J, Tang LH, Weiser MR, Brenner B, Adsay NV, Stelow EB, Saltz LB, Qin J, Landmann R, Leonard GD, Dhall D, Temple L, Guillem JG, Paty PB, Kelsen D, Wong WD, Klimstra DS. Is nonsmall cell type high-grade neuroendocrine carcinoma of the tubular gastrointestinal tract a distinct disease entity? Am J Surg Pathol. 2008;32(5):719-31. https://doi.org/10.1097/PAS.0b013e318159371c

13. Bolzacchini E, Chini C, Cortelezzi CC, Vallini I, Pinotti G, La Rosa S, Uccella S. Poorly Differentiated Neuroendocrine Carcinoma of the Sigmoid Tract in Long-Standing Ulcerative Colitis: Report of a Case and Review of the Literature. Int J Surg Pathol. 2018;26(5):479-483. https://doi.org/10.1177/1066896917752443

14. Minocha V, Shuja S, Ali R, Eid E. Large cell neuroendocrine carcinoma of the rectum presenting with extensive metastatic disease. Case Rep Oncol Med. 2014;2014:386379. https://doi.org/10.1155/2014/386379

15. Shen C, Yin Y, Chen H, Tang S, Yin X, Zhou Z, Zhang B, Chen $Z$. Neuroendocrine tumors of colon and rectum: validation of clinical and prognostic values of the World 
Health Organization 2010 grading classifications and European Neuroendocrine Tumor Society staging systems. Oncotarget. 2017;8(13):22123-22134.

https://doi.org/10.18632/oncotarget.13641

16. Choi CW, Kang DH, Kim HW, Park SB, Jo WS, Song GA, Cho M. Comparison of endoscopic resection therapies for rectal carcinoid tumor: endoscopic submucosal dissection versus endoscopic mucosal resection using band ligation. J Clin Gastroenterol. 2013;47(5):432-6. https://doi.org/10.1097/MCG.0b013e31826faf2b

17. Moertel CG, Kvols LK, O’Connell MJ, Rubin J. Treatment of neuroendocrine carcinomas with combined etoposide and cisplatin. Evidence of major therapeutic activity in the anaplastic variants of these neoplasms. Cancer. $1991 ; 68(2): 227-32$. https://doi.org/10.1002/10970142(19910715)68:2<227::aidcncr2820680202>3.0.co;2-i

18. Sun W, Lipsitz S, Catalano P, Mailliard JA, Haller DG; Eastern Cooperative Oncology Group. Phase II/III study of doxorubicin with fluorouracil compared with streptozocin with fluorouracil or dacarbazine in the treatment of advanced carcinoid tumors: Eastern Cooperative Oncology Group Study E1281. J Clin Oncol. 2005;23(22):4897-904. https://doi.org/10.1200/JCO.2005.03.616

19. Kedia S, Ahuja V, Tandon R. Management of acute severe ulcerative colitis. World J Gastrointest Pathophysiol. 2014;5(4):579-88. https://doi.org/10.4291/wjgp.v5.i4.579. PMID: 25401001

20. Yamamoto-Furusho JK, Gutiérrez-Grobe Y, LópezGómez JG, Bosques-Padilla F, Rocha-Ramírez JL; Grupo del Consenso Mexicano de Colitis Ulcerosa Crónica Idiopática. Consenso mexicano para el diagnóstico y tratamiento de la colitis ulcerosa crónica idiopática. Rev Gastroenterol Mex. 2018;83(2):144-167. https://doi.org/10.1016/j.rgmx.2017.08.006

21. Li Y, Shen B. Evaluating pouch problems. Gastroenterol Clin North Am. 2012;41(2):355-78. https://doi.org/10.1016/j.gtc.2012.01.013

22. Gallone L, Olmi L, Marchetti V. Use of topical rectal therapy to preserve the rectum in surgery of ulcerative colitis. World J Surg. 1980;4(5):609-13. https://doi.org/10.1007/BF02401644

23. Trujillo-Díaz JJ, Blanco-Antona F, de Solórzano-Aurusa JO, Martínez-García G, Fernández-Salazar L, Rentería JPBHY. Neuroendocrine tumor associate with inflammatory bowel disease: two cases report. Cir Cir. 2019;87(S1):17-21. https://doi.org/10.24875/CIRU.18000519

24. La Rosa S, Klersy C, Uccella S, Dainese L, Albarello L, Sonzogni A, Doglioni C, Capella C, Solcia E. Improved histologic and clinicopathologic criteria for prognostic evaluation of pancreatic endocrine tumors. Hum Pathol. 2009;40(1):30-40. https://doi.org/10.1016/j.humpath.2008.06.005

25. Sánchez-Bueno F, Rodríguez González JM, Torres Salmerón G, Bernabé Peñalver A, Balsalobre Salmeron M, de la Peña Moral J, Fuster Quiñonero M, Parrilla Paricio P. Prognostic factors in resected pancreatic neuroendocrine tumours: Experience in 95 patients. Cir Esp. 2016;94(8):473-80. https://doi.org/10.1016/j.ciresp.2016.05.010 